\section{Uso do chumbo em urina como indicador de exposição e sua relação com chumbo no sangue}

\author{
Use of urine lead level as an exposure indicator \\ and its relationship to blood lead
}

\begin{abstract}
The aim of this work was to verify whether there are statistically significant correlation between the concentrations of lead in blood $(\mathrm{Pb}-\mathrm{B})$ and urine $(\mathrm{Pb}-\mathrm{U})$. Electrothermal atomic absorption spectrometry was used in the determination of lead concentration in biological material. Venous blood and spot urine were collected from workers occupationally exposed (95), adults (130) and children up to 15 years old (22) environmentally exposed. After a test showing significant differences between $\mathrm{Pb}$-U and the three categories previously determined, cutting points for $\mathrm{Pb}$-U were established to predict $\mathrm{Pb}-\mathrm{B}$ values by the ROC curve. Thus, it is expected that $P b-B$ is lower than $10 \mu \mathrm{g}$.dL-1 with $\mathrm{Pb}-U$ up to $0.55 \mu \mathrm{g}$. $d L-1$, whereas lead levels in blood below $27.6 \mu \mathrm{g}$. $d L^{-1}$ are expected when the amount of the metal in urine is lower than $2.05 \mu \mathrm{g} . d L-1$. So, urine can be used to replace blood for the assessment of the occupational exposure to lead. However, caution is advised in the case of environmental exposure, since urinary lead should be used just as an estimation of the metal content in blood.
\end{abstract}

Lead; Occupational Exposure; Blood; Urine
Maria de Fátima Ramos Moreira 1

Eduardo Borba Neves 1

\section{Introdução}

O chumbo $(\mathrm{Pb})$ é um metal tóxico de ocorrência natural na crosta terrestre e sem nenhuma função fisiológica no organismo humano. Entretanto, seus efeitos nocivos são conhecidos desde os tempos antigos, por afetarem praticamente todos os órgãos e sistemas do corpo humano ${ }^{1}$.

No organismo, esse elemento não é metabolizado, e, sim, complexado por macromoléculas, sendo diretamente absorvido, distribuído e excretado. Uma vez absorvido, o chumbo se distribui entre o sangue, os tecidos moles (rins, medula óssea, fígado e cérebro) e os tecidos mineralizados (ossos e dentes) 2.

A retenção do chumbo nos tecidos moles se estabiliza na vida adulta, podendo até decrescer em alguns órgãos com o avanço da idade. Contudo, continua a se acumular nos ossos e na aorta durante toda a vida. Os tempos de meia-vida do metal são bastante diferentes, sendo estimados em 36 dias para o sangue, 40 dias para os tecidos moles e 27 anos para os ossos 2,3.

O chumbo é excretado por várias rotas, porém só a excreção renal e a gastrintestinal têm importância prática. A quantidade excretada é afetada pela idade, características da exposição e dependente da espécie ${ }^{4}$. Entre crianças e adultos, existem inúmeras diferenças relacionadas à idade, e a comparação dos dados sobre a cinética do chumbo em ambas as fases mostra que, aparentemente, as primeiras parecem ter uma 
menor taxa total de excreção. Crianças até dois anos de idade retêm $34 \%$ da quantidade total de chumbo absorvido, ao passo que esta retenção é de apenas $1 \%$ nos adultos 5,6.

Os efeitos biológicos do chumbo são os mesmos, independentemente das rotas de entrada, uma vez que ele interfere no funcionamento normal da célula e em inúmeros processos fisiológicos. Nas crianças, esses efeitos atingem principalmente o sistema nervoso, enquanto, nos adultos, os cuidados são com a neuropatia periférica e a nefropatia crônica 7 .

Apesar de os indicadores de dose interna, tais como chumbo em sangue total, plasma e urina, serem muito utilizados, todos têm algum tipo de limitação ${ }^{8}$.Porisso, a concentração de chumbono sangue total ( $\mathrm{Pb}-\mathrm{S}$ ) ainda é aceita como indicador de exposição total a esse elemento, embora indique uma exposição ambiental recente ${ }^{9}$. Por sua vez, a concentração de chumbo na urina (Pb-U) tem sido aplicada como teste de exposição na saúde ocupacional, já que a medida deste parâmetro reflete exposição atual 3 . A urina é um indicador de exposição potencialmente útil, porém a relação existente entre o chumbo no sangue e na urina ainda não é perfeitamente compreendida 10 .

Apesar de o chumbo no sangue ser utilizado como medida padrão no monitoramento biológico da exposição ocupacional a este metal, as experiências no campo reforçam a idéia de que freqüentemente a amostragem de sangue não é uma atividade de fácil realização. Crianças e, até mesmo, alguns adultos têm dificuldades em permitir a coleta por inúmeras razões, tais como: medo, presença de tecido adiposo ao redor das veias, entre outras. Assim, seria desejável que, na prática da saúde ocupacional e ambiental, o chumbo na urina pudesse ser uma alternativa válida para o chumbo no sangue.

Embora existam inúmeros estudos sobre a contaminação por chumbo 11,12,13,14,15,16,17,18, 19,20,21,22,23,24, poucos são aqueles que descrevem as relações entre o metal no sangue e na urina 8,16, $17,18,19,20,21,22,23,24$. Uma revisão sobre biomarcadores de exposição ao chumbo relata a existência de correlação não linear entre esses dois indicadores biológicos ${ }^{8}$. Por outro lado, pesquisas com trabalhadores expostos a esse elemento encontram forte correlação entre o chumbo no sangue e na urina $16,18,21$. No caso de exposição ambiental, o Pb-U não pode ser usado em substituição ao $\mathrm{Pb}-\mathrm{S}$ de acordo com os resultados de estudos realizados com a população em geral 17,19,22. Uma investigação, da qual crianças também fizeram parte, concluiu que a concentração de chumbo na urina não pode ser usada para predizer o teor do metal no sangue, particularmente nos níveis mais baixos de exposição, por exemplo, com $\mathrm{Pb}-\mathrm{S}$ menor do que $10 \mu \mathrm{g} . \mathrm{dL}^{-1} 17$.

Portanto, o objetivo deste estudo foi verificar se há correlação entre as concentrações de chumbo no sangue e na urina de crianças e adultos com diferentes níveis de exposição, visando à substituição do sangue pela urina como indicador de exposição, por utilizar um método de coleta não invasivo.

\section{Experimental}

\section{Instrumental}

Um espectrômetro de absorção atômica 5.100 com efeito Zeeman como corretor de fundo, forno de grafite HGA-600 e amostrador automático AS60, todos Perkin-Elmer (Perkin-Elmer Norwalk, Estados Unidos), foram utilizados neste trabalho. O comprimento de onda da lâmpada de catodo oco (Perkin-Elmer) de chumbo foi de 283,3 nm e a largura da fenda foi de $0,7 \mathrm{~nm}$. Tubos recobertos com grafite pirolítico (Perkin-Elmer, cat.no. B0109322) e plataformas de L'vov (Perkin-Elmer, cat. $n^{\circ}$. B0109324) foram usados em todos os experimentos. O programa de temperatura utilizado para a determinação de chumbo no sangue e na urina pode ser encontrado na Tabela 1 .

\section{Coleta e armazenamento das amostras}

As amostras de sangue foram coletadas em tubos a vácuo heparinizados, específicos para a determinação de elementos-traço. Já as de urina foram coletadas diretamente em frascos de polietileno previamente descontaminados. Ambos os materiais biológicos foram congelados até posterior análise.

\section{Materiais, reagentes e amostras}

Toda a vidraria e utensílios plásticos utilizados ficaram imersos em uma solução de Extran (Merck, Elmsford, Estados Unidos) a 5\% (v/v), por um período mínimo de 24 horas. Após este tempo, o material era enxaguado com água corrente em abundância e, em seguida, imerso em uma solução de ácido nítrico a 10\% (v/v) para a descontaminação por, pelo menos, 48 horas. Após ser enxaguado várias vezes com água deionizada previamente purificada pelo sistema Milli-Q (Millipore, Bedford, Estados Unidos), o material era seco em estufa a $30^{\circ} \mathrm{C}$.

Todos os reagentes utilizados foram, pelo menos, de grau analítico (P.A.). As soluções analíticas de chumbo eram preparadas diariamente em ácido nítrico 0,2\% (v/v) (Merck) por diluições 
Programa de temperatura para determinação de chumbo em sangue e urina.

\begin{tabular}{lcccc}
\hline Etapa & Temperatura $\left({ }^{\circ} \mathrm{C}\right)$ & Rampa (s) & "Hold" (s) & Fluxo de ar (mL.min-1) \\
\hline $1^{*}$ & 90 & 1 & 10 & 300 \\
$2^{* *}$ & 120 & 10 & 20 & 300 \\
3 & 1.100 & 10 & 20 & 300 \\
4 & 20 & 1 & 10 & 300 \\
$5 * \star *$ & 1.900 & 0 & 5 & 0 \\
6 & 2.600 & 1 & 3 & 300 \\
7 & 20 & 1 & 5 & 300 \\
\hline
\end{tabular}

* Modificador:

** Amostra;

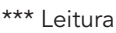

adequadas da solução estoque de $1.000 \mu \mathrm{g} \cdot \mathrm{mL}^{-1}$ de chumbo preparada a partir do concentrado Titrisol-Merck.

Na preparação do modificador, foi utilizada uma mistura de nitrato de magnésio $10 \mathrm{gL}^{-1}$ e nitrato de paládio 10 g.L-1, também Merck, diluída em ácido nítrico $0,2 \%(\mathrm{v} / \mathrm{v})$, de modo a conter $10 \mu \mathrm{g}$ de nitrato de magnésio e $15 \mu \mathrm{g}$ de paládio metálico em $10 \mu \mathrm{L}$ de solução dentro do forno.

O sangue total foi diluído em Triton X-100 $0,1 \%(\mathrm{v} / \mathrm{v})$ (Merck), na proporção de $1+9$ para amostras de pessoas expostas ambientalmente, enquanto uma diluição de vinte vezes foi utilizada no sangue de trabalhadores expostos ocupacionalmente. Já a urina foi diluída na proporção de $1+4$ em ácido nítrico $0,2 \%(\mathrm{v} / \mathrm{v})$.

A qualidade analítica dos resultados foi monitorada por intermédio de programas de controle interlaboratorial com o Instituto Nacional de Seguridad e Higiene en el Trabajo (Zaragoza, Espanha) e Instituto Adolfo Lutz (São Paulo, Brasil). A exatidão dos resultados também foi acompanhada por meio da análise, em cada série de amostras, dos seguintes materiais de referência, diluídos conforme sua concentração: (a) Contox Heavy Metal Blood Control (Kaulson Laboratories, Estados Unidos): $20 \pm 4 \mu \mathrm{g} . \mathrm{dL}^{-1}$; (b) Contox Blood Lead Control (Kaulson Laboratories, Estados Unidos): $5 \pm 3 \mu \mathrm{g} . \mathrm{dL}^{-1}$ (low level), 25 - $4 \mu \mathrm{g} \cdot \mathrm{dL}^{-1}$ (medium level) e $52 \pm 5 \mu \mathrm{g} . \mathrm{dL}^{-1}$ (high level); (c) Lyphochek Urine Metals Control (BIO-RAD, Estados Unidos): 14,7 - 3,0 $\mu$ g.L-1 (level 1) e 72 - 14,5 $\mu$ g.L-1 (level 2); (d) Toxic Metals in Freeze-Dried Urine SRM 2670 (NIST, Estados Unidos): $10 \mu \mathrm{g} . \mathrm{L}^{-1}$ (low level) e $109 \pm 4 \mu \mathrm{g} . \mathrm{L}^{-1}$ (elevated level).

\section{Metodologia}

Esta pesquisa utilizou os resultados de 247 análises de sangue e urina realizadas pelo Setor de Metais do Laboratório de Toxicologia do Centro de Estudos da Saúde do Trabalhador e Ecologia Humana, Escola Nacional de Saúde Pública Sergio Arouca, Fundação Oswaldo Cruz (CESTEH/ ENSP/FIOCRUZ). Os dados relativos ao monitoramento biológico de diversos grupos populacionais, participantes de diferentes projetos sobre a avaliação da exposição ao chumbo entre os anos de 1994 e 2005, permitiram a construção desse banco. Todos os sujeitos avaliados assinaram o Termo de Consentimento Livre e Esclarecido, aplicado pelos integrantes dos projetos, assim como preencheram um questionário para obtenção de informações que permitissem conhecer os pacientes e seus hábitos. Ambos foram aprovados pelos Comitês de Ética em Pesquisa da ENSP/ FIOCRUZ.

As amostras de sangue venoso e de urina pontual foram coletadas entre trabalhadores expostos ocupacionalmente (95), pessoas expostas ambientalmente (130) e crianças até 15 anos (22) também expostas ambientalmente. Essas pessoas foram categorizadas de acordo com o nível de exposição, com exceção das crianças, que formaram uma classe à parte, por apresentarem metabolismo ósseo diferente daquele dos adultos.

As categorias foram determinadas após a escolha de dois pontos de corte, baseados na experiência do setor, com resultados da concentração de chumbo no sangue de indivíduos com diferentes situações de exposição ao metal, e também em concordância com o limite superior de 27,6 $\mu$ g.dL-1 para o valor de referência estabelecido 
pela Comunidade Européia ${ }^{25}$. Assim, a faixa até $10 \mu \mathrm{gPb} . \mathrm{dL}^{-1}$ no sangue foi considerada como exposição ambiental, entre $10 \mu \mathrm{g} \cdot \mathrm{dL}^{-1}$ e $27,6 \mu \mathrm{g} \cdot \mathrm{dL}^{-1}$ classificou-se como exposição ocupacional moderada.e acima de 27,6 $\mu$ g.dL-1 , como exposição ocupacional elevada. Todo o tratamento estatístico dos dados foi realizado com o SPSS versão 13.0 (SPSS Inc., Chicago, Estados Unidos).

\section{Resultados e discussão}

Inicialmente, o teste de Kolmogorov-Smirnov foi aplicado às variáveis $\mathrm{Pb}-\mathrm{U}$ e $\mathrm{Pb}$-S em todo o grupo, separadas por categorias, a fim de verificar se seguiam uma distribuição normal. O resultado significativo mostrou que esse tipo de distribuição não era uma boa aproximação para essas variáveis. Neste caso, correlações não paramétricas devem ser aplicadas; assim, foi utilizada a correlação de Spearman. Também Schutz et al. 16 concluíram que as variáveis sob análise não seguiam a distribuição normal, por conseguinte aplicaram as estatísticas não paramétricas.

A relação entre $\mathrm{Pb}-\mathrm{S}$ e $\mathrm{Pb}-\mathrm{U}$ foi analisada nas quatro categorias criadas (exposição ocupacional elevada, exposição ocupacional moderada, exposição ambiental e crianças). Os resultados encontrados mostram correlações moderadas positivas para os três tipos de exposição, todas significativas $(p<0,05)$. Entretanto, o grupo de crianças apresentou uma correlação negativa ( $p=0,104)$ sem significância estatística. Esses resultados podem ser encontrados na Tabela 2, enquanto o comportamento de $\mathrm{Pb}-\mathrm{S}$ e $\mathrm{Pb}-\mathrm{U}$ para o grupo das crianças pode ser observado na Figura 1. Os valores médios e os respectivos desviospadrão (DP) de chumbo no sangue e na urina das diferentes categorias também são mostrados na Tabela 2.

Essa correlação negativa pode ter sido influenciada pela incapacidade analítica instrumental em medir concentrações de $\mathrm{Pb}-\mathrm{U}$ abaixo do limite de detecção (LOD) de 0,40 $\mu$ g.dL-1, pois, nesse grupo, aproximadamente $61 \%$ dos resultados encontrados para este indicador foram iguais ou menores do que o LOD. Desta forma, os sujeitos com teores inferiores a esse valor têm a mesma representatividade do que aqueles cuja concentração de $\mathrm{Pb}-\mathrm{U}$ realmente seja $0,40 \mu \mathrm{g} \cdot \mathrm{dL}^{-1}$, diminuindo, assim, a confiabilidade da análise estatística.

Como crianças e adultos apresentam respostas fisiológicas diferentes à exposição ao chumbo, uma outra análise de correlação total foi realizada excluindo as crianças. Dessa vez, uma forte correlação foi encontrada entre os indicadores $\mathrm{Pb}-\mathrm{S}$ e $\mathrm{Pb}-\mathrm{U}(\mathrm{r}=0,823$; com $\mathrm{p}=0,000)$, porém com grande variância na razão entre eles, dificultando, dessa forma, a predição dos valores de $\mathrm{Pb}-\mathrm{S}$ em função de $\mathrm{Pb}-\mathrm{U}$.

Os resultados encontrados nesta pesquisa para as correlações entre $\mathrm{Pb}-\mathrm{U}$ e $\mathrm{Pb}-\mathrm{S}$ estão de acordo com aqueles relatados em outros estudos realizados com crianças e adultos expostos ambiental e ocupacionalmente ao chumbo $16,17,18,19,20,21,22,23,24$. Um estudo sobre mulheres e crianças expostas ambientalmente encontrou uma relação linear pobre entre os dois indicadores pesquisados, particularmente nas faixas mais baixas de exposição ${ }^{17}$. Shimbo et al. 19 examinaram a correlação entre os resultados de chumbo no sangue e na urina de mulheres adultas não fumantes através da análise de regressão e concluíram que o uso de $\mathrm{Pb}-\mathrm{U}$ em lugar de $\mathrm{Pb}-\mathrm{S}$ não era confiável, uma vez que a correlação encontrada era significante, porém fraca. Amostras de sangue e urina de mulheres expostas ambientalmente foram analisadas, e a correlação entre $\mathrm{Pb}-\mathrm{S}$ e $\mathrm{Pb}-\mathrm{U}$ foi significativa em todos os casos estudados, quando o nível de $\mathrm{Pb}-\mathrm{S}$ era maior ou igual a $50 \mu \mathrm{g} . \mathrm{L}^{-1}$. Dessa forma, os autores concluíram que $\mathrm{Pb}-\mathrm{S}$ poderia ser estimado a partir de $\mathrm{Pb}-\mathrm{U}$, tendo o grupo como base 20 .

Uma outra pesquisa com mulheres de meiaidade também encontrou fraca correlação entre o chumbo na urina e no sangue e concluiu que o $\mathrm{Pb}-\mathrm{U}$ não deveria ser usado como marcador de exposição ${ }^{22}$. A relação entre o teor de chumbo no sangue total corrigido pelo hematócrito e a urina de 24 horas foi examinada em amostras de homens de meia-idade e de idade avançada, sem exposição ocupacional ao chumbo. Os resultados mostraram que $\mathrm{Pb}-\mathrm{S}$ e $\mathrm{Pb}-\mathrm{U}$ permaneciam significativamente associados 24 .

Em um estudo publicado recentemente, uma forte correlação foi encontrada entre $\mathrm{Pb}-\mathrm{U}$ e $\mathrm{Pb}-\mathrm{S}$ $(\mathrm{r}=0,78 ; \mathrm{p}<0,001)$ de trabalhadores aposentados e da ativa de uma fundição de chumbo na Alemanha 16. Resultado semelhante $(r=0,824 ; \mathrm{p}<0,01)$ foi encontrado em outra pesquisa realizada com população ocupacionalmente exposta, que sugere o uso de $\mathrm{Pb}-\mathrm{U}$ como uma boa alternativa ao $\mathrm{Pb}-\mathrm{S}$, com base em grupos. No entanto, adverte que essa correlação não foi próxima o suficiente para permitir a predição de $\mathrm{Pb}-\mathrm{S}$ em bases individuais 18 . Hirata et al. ${ }^{21}$ constataram que $\mathrm{Pb}-\mathrm{S}$ e $\mathrm{Pb}-\mathrm{U}$ se correlacionavam significativamente em trabalhadores expostos ao chumbo, porém o coeficiente de correlação entre a concentração do metal na urina e no plasma foi sempre maior do que aquele entre urina e sangue em qualquer nível de exposição. Uma boa correlação $(r=$ 0,78 ) também foi encontrada entre o chumbo sanguíneo e urinário de trabalhadores expostos ao metal em fundições na Suécia e Alemanha. 
Tabela 2

Valor médio, desvio-padrão (DP) e correlação (r) entre concentração de chumbo no sangue total (Pb-S) concentração de chumbo na urina $(\mathrm{Pb}-\mathrm{U})(\mu \mathrm{g} \cdot \mathrm{dL}-1)$ total e por categoria.

\begin{tabular}{|c|c|c|c|c|c|}
\hline Tipo de exposição & $\begin{array}{c}\mathrm{Pb}-\mathrm{S} \\
\text { Média } \pm \mathrm{DP}\end{array}$ & $\begin{array}{c}\mathrm{Pb}-\mathrm{U} \\
\text { Média } \pm \mathrm{DP}\end{array}$ & $\mathbf{r}$ & Valor de $p$ & $\mathrm{n}$ \\
\hline Total & $22,3 \pm 32,2$ & $3,6 \pm 7,6$ & 0,771 & 0,000 & 247 \\
\hline Ocupacional elevada & $67,6 \pm 38,1$ & $12,0 \pm 11,9$ & 0,482 & 0,004 & 61 \\
\hline Ocupacional moderada & $17,9 \pm 4,4$ & $2,0 \pm 1,6$ & 0,526 & 0,000 & 34 \\
\hline Ambiental & $5,4 \pm 2,1$ & $0,6 \pm 0,4$ & 0,322 & 0,000 & 130 \\
\hline Crianças & $5,8 \pm 2,6$ & $0,5 \pm 0,2$ & $-0,356$ & 0,104 & 22 \\
\hline
\end{tabular}

Figura 1

Concentração de chumbo no sangue $(\mathrm{Pb}-\mathrm{S})$ e na urina $(\mathrm{Pb}-\mathrm{U})$ de crianças.

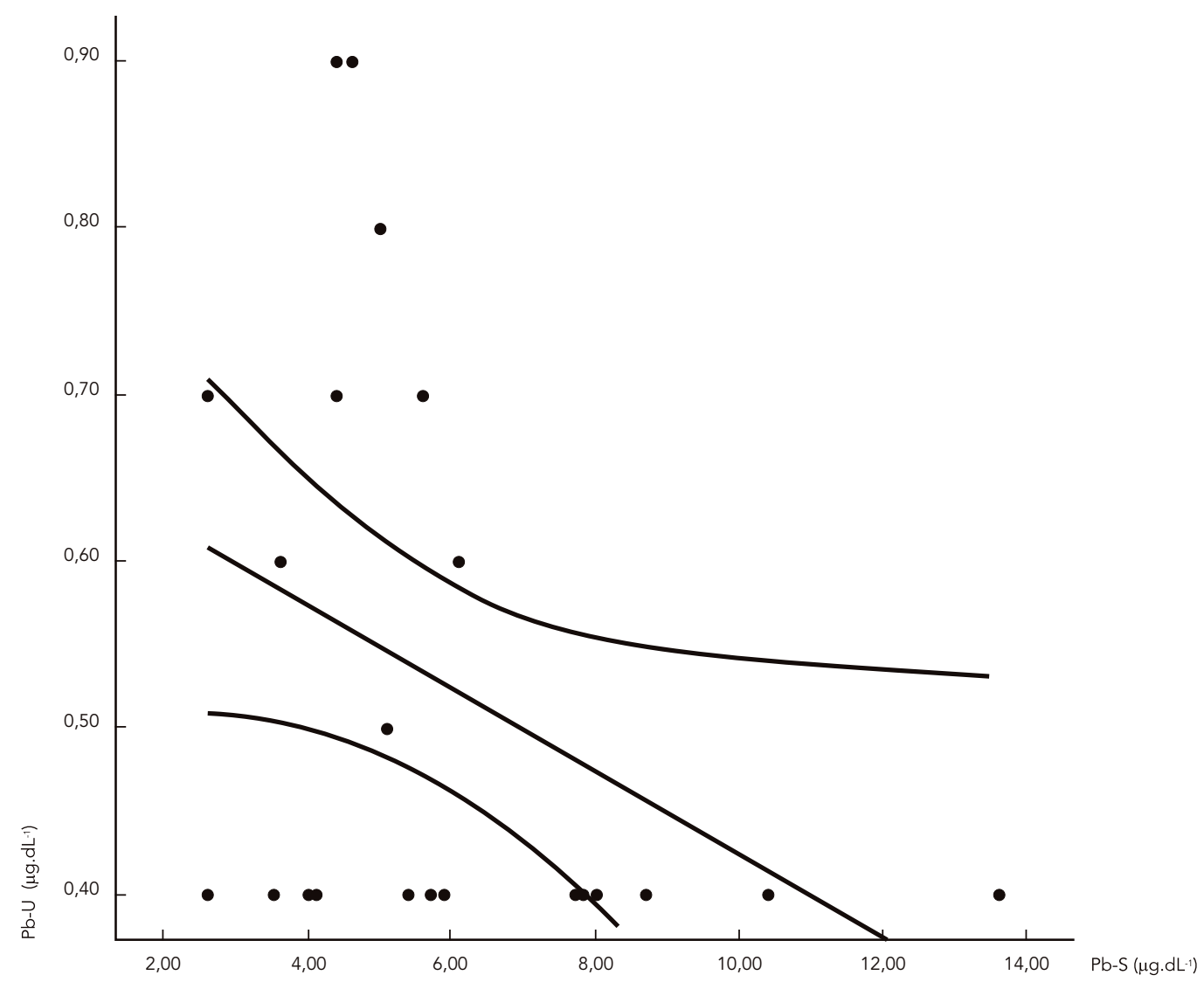


Contudo, esta relação mostrou grande dispersão, parecendo ser exponencial 23 .

A aplicação do teste de Mann-Whitney mostrou a existência de diferenças significativas entre os resultados de $\mathrm{Pb}-\mathrm{U}$ das três categorias previamente determinadas. Tal fato indica a possibilidade da definição de pontos de corte para esse indicador. Assim, os pontos de corte para $\mathrm{Pb}-\mathrm{U}$ foram calculados a partir daqueles já estabelecidos para $\mathrm{Pb}-\mathrm{S}$, por meio da curva ROC (Receiver Operating Characteristics), conforme Figuras 2 e 3.

A comparação das curvas de diferentes testes diagnósticos é uma das vantagens deste método. A curva ROC também é a maneira mais adequada de estabelecer pontos de corte, melhorando a sensibilidade e a especificidade do teste diagnóstico 26 . Em relação ao gráfico, quanto mais próxi- ma a curva estiver do canto superior esquerdo, melhor será o teste. Ao construir essa curva, o programa SPSS apresenta uma tabela com valores de $\mathrm{Pb}-\mathrm{U}$, respectivas sensibilidades e complementos da especificidade.

A partir das tabelas geradas juntamente com as Figuras 2 e 3, os valores 0,55 $\mu$ g.dL-1 e 2,15 $\mu \mathrm{g} . \mathrm{dL}^{-1}$ foram selecionados como pontos de corte para o indicador $\mathrm{Pb}-\mathrm{U}$, privilegiando-se a sensibilidade do indicador. Assim, concentrações de chumbo na urina até $0,55 \mu \mathrm{g} . \mathrm{dL}^{-1}$ correspondem a níveis de $\mathrm{Pb}-\mathrm{S}$ menores do que $10 \mu \mathrm{g} \cdot \mathrm{dL}^{-1} \mathrm{com}$ sensibilidade de 97,9\% ( $p=0,000)$, especificidade de $64,4 \%$ ( $p=0,000$ ), valor preditivo positivo de $97,7 \%$ e valor preditivo negativo de $65,7 \%$. Foi possível estabelecer também que, para teores de $\mathrm{Pb}-\mathrm{U}$ até $2,15 \mu \mathrm{g} . \mathrm{dL}^{-1}$, pode-se esperar que os valores de $\mathrm{Pb}-\mathrm{S}$ sejam menores que 27,6 $\mu$ g.dL $\mathrm{dL}^{-1}$

Figura 2

Curva ROC (Receiver Operating Characteristics) para valores de concentração de chumbo na urina (Pb-U) com corte em concentração de chumbo no sangue total $\left(\mathrm{Pb}-\mathrm{S}=10 \mu \mathrm{g} \cdot \mathrm{dL}^{-1}\right)$.

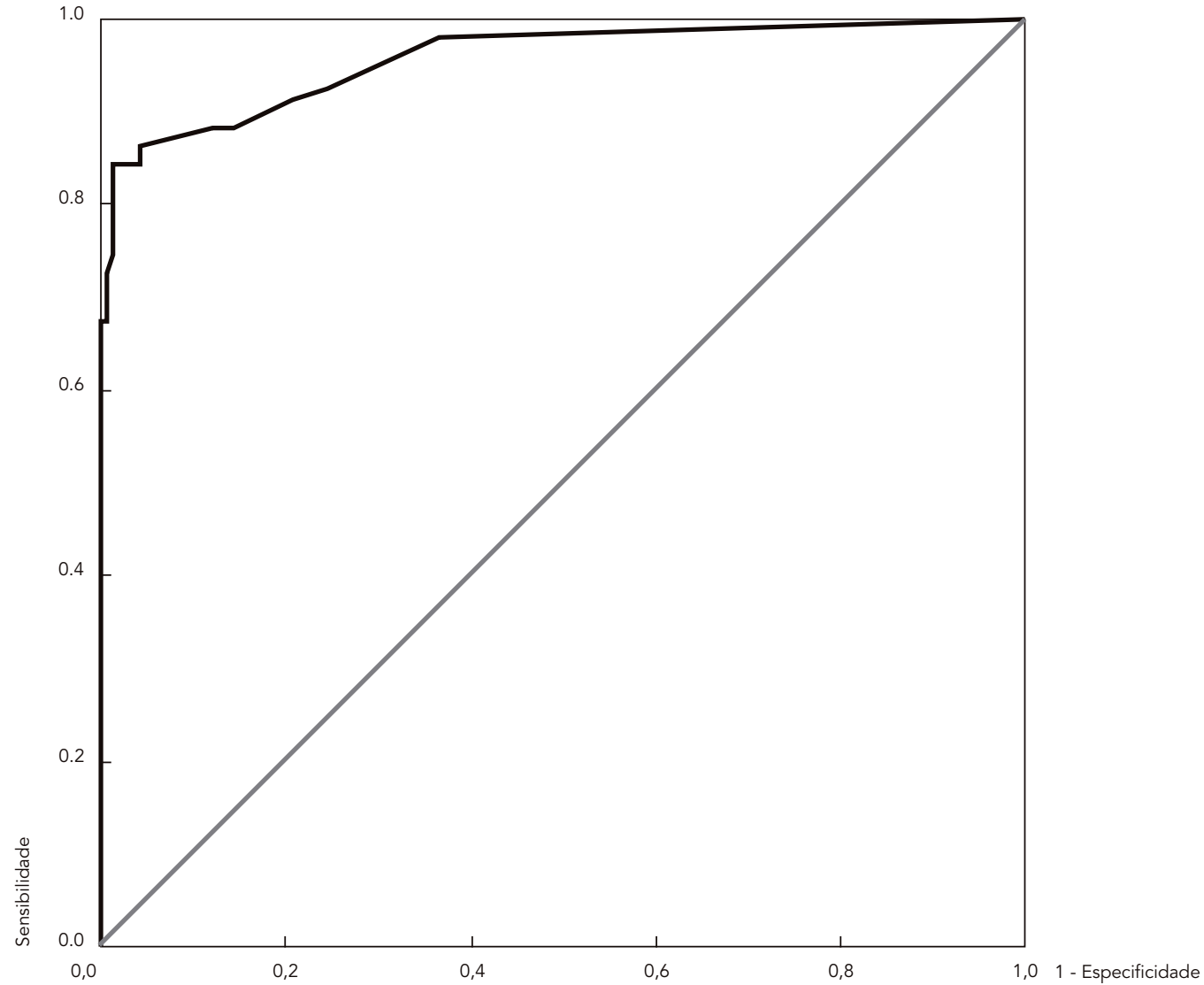


Curva ROC (Receiver Operating Characteristics) para valores de concentração de chumbo na urina (Pb-U) com corte em concentração de chumbo no sangue total ( $\mathrm{Pb}-\mathrm{S}=27,6 \mu \mathrm{g} \cdot \mathrm{dL}-1)$.

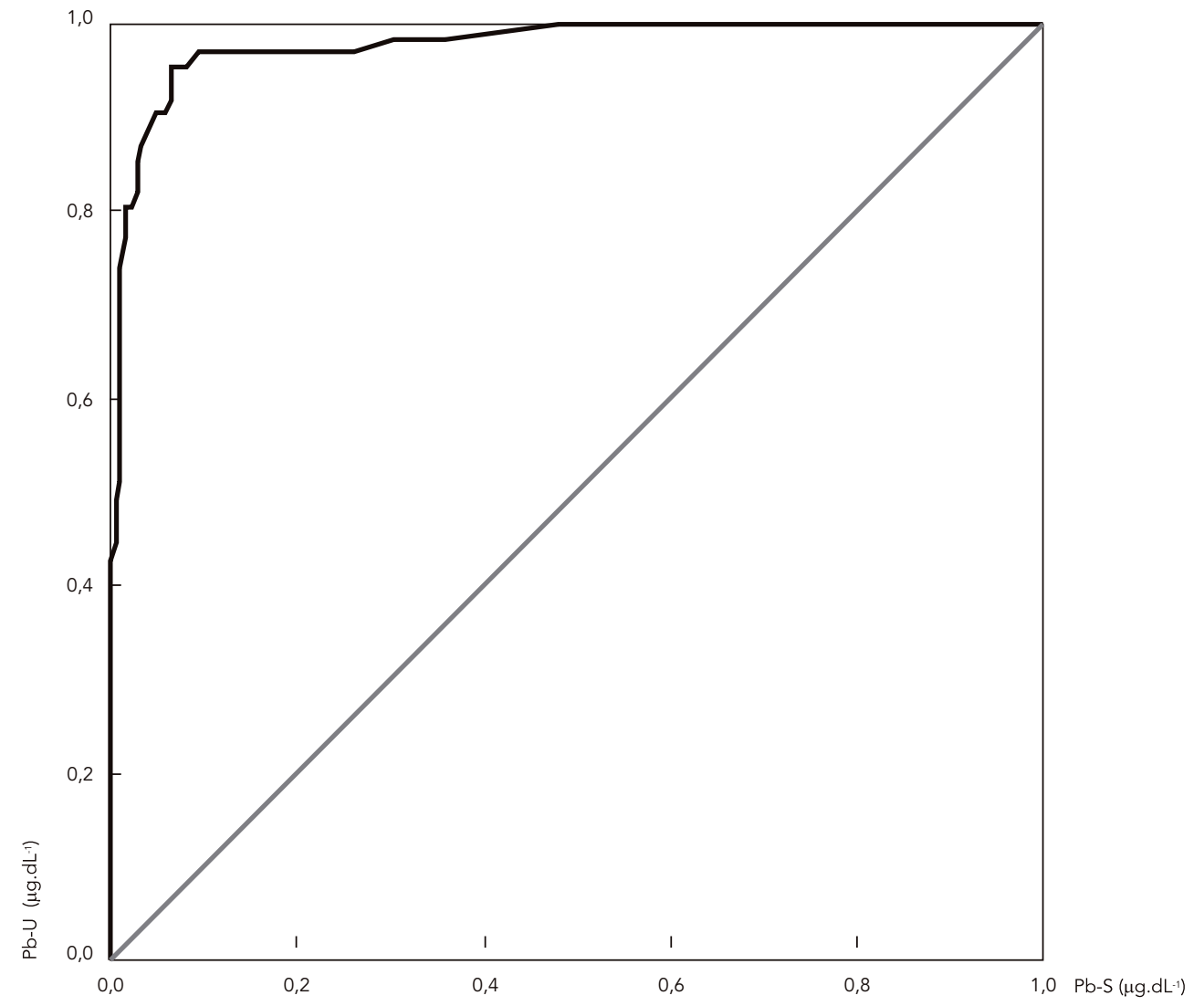

com sensibilidade de $95,1 \%$ ( $p=0,000)$, especificidade de $93,3 \%$ ( $p=0,000)$, valor preditivo positivo de $99,4 \%$ e valor preditivo negativo de $84,1 \%$.

Dessa forma, indivíduos com valores de $\mathrm{Pb}-\mathrm{U}$ menores do que $0,55 \mu \mathrm{g} \cdot \mathrm{dL}^{-1}$ podem ser classificados como expostos ambientalmente, ao passo que aqueles que apresentarem $\mathrm{Pb}-\mathrm{U}$ abaixo de 2,15 $\mu$ g.dL-1 farão parte da categoria de exposição ocupacional moderada; conseqüentemente, o grupo com exposição ocupacional elevada apresentará $\mathrm{Pb}-\mathrm{U}$ acima de 2,15 $\mu \mathrm{g} . \mathrm{dL}^{-1}$.

Os valores preditivos positivos retratam a probabilidade de um sujeito com $\mathrm{Pb}-\mathrm{U}$ menor do que $0,55 \mu$ g.dL-1 ou 2,15 $\mu$ g.dL-1 ter um valor de $\mathrm{Pb}-\mathrm{S}$ menor do que $10 \mu \mathrm{g} . \mathrm{dL}^{-1}$ ou 27,6 $\mu$ g.dL-1, respectivamente. Por outro lado, as chances de indivíduos com $\mathrm{Pb}$-U maiores do que $0,55 \mu \mathrm{g} . \mathrm{dL}^{-1}$ ou 2,15 $\mu$ g.dL $\mathrm{dL}^{-1}$ apresentarem teores de chumbo no sangue acima dos pontos de corte estabelecidos (10 $\mu$ g.dL-1 ou 27,6 $\mu$ g.dL-1) são ditadas pelos valores preditivos negativos.

Ambos os valores preditivos positivos foram bem próximos de $100 \%$, mostrando que as afirmações são confiáveis, até mesmo no grupo dos expostos ambientalmente, cuja correlação entre os indicadores foi a mais fraca. A faixa de concentração abaixo de 2,15 $\mu$ g.dL-1 para $\mathrm{Pb}-\mathrm{U}$ apresentou um valor preditivo negativo elevado $(84,1 \%)$, enquanto aquela com níveis de chumbo em urina menores do que $0,55 \mu \mathrm{g} \cdot \mathrm{dL}^{-1}$ se limitou a $65,7 \%$. Assim, as chances de os expostos moderadamente apresentarem um $\mathrm{Pb}-\mathrm{S}$ maior do que 27,6 $\mu$ g.dL-1 são maiores do que aquelas da categoria com exposição ambiental.

Apesar da utilização sistemática dos índices de correlação estatística nos trabalhos que investigam a relação entre $\mathrm{Pb}-\mathrm{U}$ e $\mathrm{Pb}-\mathrm{S}$, não foram 
encontradas publicações que tenham feito uso da curva ROC para predição de $\mathrm{Pb}-\mathrm{S}$ a partir de $\mathrm{Pb}-\mathrm{U}$.

\section{Conclusão}

O grupo das crianças apresentou uma correlação negativa, sem significância estatística, impossibilitando para este grupo a substituição do sangue pela urina como indicador biológico de exposição ao chumbo. Todavia, correlações moderadas positivas, todas significativas, foram encontradas para as outras três categorias. As duas classes relativas à exposição ocupacional apresentaram correlações ainda melhores do que o grupo de expostos ambientalmente.

Apesar da forte correlação, não é aconselhável a utilização do $\mathrm{Pb}$-U como preditor direto dos valores de $\mathrm{Pb}$-S, em virtude da grande variabilidade das razões dos indicadores inter-individuais. En- tretanto, a utilização do $\mathrm{Pb}-\mathrm{U}$ como teste para avaliação da contaminação de trabalhadores por chumbo tem como vantagem evitar que estes sejam submetidos a exames invasivos periódicos, uma vez que esse tipo de amostra não traz desconforto ao trabalhador e apresenta maior facilidade na coleta, armazenamento, transporte e manuseio.

O estabelecimento de pontos de corte para valores de $\mathrm{Pb}-\mathrm{U}$ pode ajudar no levantamento dos trabalhadores expostos ao chumbo e contribuir de forma concreta para a simplificação do monitoramento biológico, usado na vigilância da saúde do trabalhador. Portanto, a urina pode ser utilizada em substituição ao sangue para avaliação da exposição ocupacional a este metal. Contudo, no caso da exposição ambiental, recomenda-se cautela nessa substituição, devendose utilizar o chumbo urinário como uma estimativa do conteúdo do metal no sangue.

\section{Resumo}

O objetivo deste trabalho foi verificar se há correlação estatisticamente significativa entre as concentrações de chumbo no sangue ( $\mathrm{Pb}$-S) e urina ( $\mathrm{Pb}$-U). A espectrometria de absorção atômica eletrotérmica foi utilizada na determinação da concentração de chumbo no material biológico. As amostras de sangue e de urina foram coletadas entre trabalhadores expostos ocupacionalmente (95) e entre adultos (130) e crianças até 15 anos (22) expostos ambientalmente. Após um teste que mostrou diferenças significativas entre $\mathrm{Pb}-\mathrm{U}$ e as três categorias previamente determinadas, pontos de corte em $\mathrm{Pb}$-U puderam ser fixados para a predição dos valores de Pb-S pela curva ROC. Assim, para Pb-U até 0,55 $\mu \mathrm{g} . d L^{-1}$, pode-se esperar que $P b$-S seja menor do que $10 \mu \mathrm{g} . d L^{-1}$, ao passo que níveis de chumbo no sangue até $27,6 \mu \mathrm{g} . d L^{-1}$ são esperados quando o teor do metal na urina é menor do que 2,05 $\mu \mathrm{g} . d L^{-1}$. Logo, a urina pode ser utilizada em substituição ao sangue para avaliação da exposição ocupacional ao chumbo. Contudo, recomenda-se cautela no caso da exposição ambiental, devendo-se utilizar o chumbo urinário como uma estimativa do conteúdo do metal no sangue.

Chumbo; Exposição Ocupacional; Sangue; Urina

\section{Colaboradores}

M. F. R. Moreira trabalhou na concepção, na metodologia e na redação final do artigo. E. B. Neves contribuiu na análise estatística, discussão e na redação final. 


\section{Referências}

1. Xie Y, Chiba M, Shinohara A, Watanabe H, Inaba Y. Studies on lead-binding protein and interaction between lead and selenium in the human erythrocytes. Ind Health 1998; 36:234-9.

2. Tsalev DL, Zaprianov ZK. In: Atomic absorption spectrometry in occupational and environmental health practice. $2^{\text {nd }}$ Ed. Boca Raton: CRC Press; 1985.

3. Moreira FR, Moreira JC. A cinética do chumbo no organismo humano e sua importância para a saúde. Ciênc Saúde Coletiva 2004; 9:167-81.

4. World Health Organization. Environmental health criteria 165: inorganic lead. Geneva: World Health Organization; 1995.

5. U.S. Department of Health and Human Services, Public Health Service, Agency for Toxic Substances and Disease Registry. Toxicological profile for lead. Atlanta: U.S. Department of Health and Human Services; 1999.

6. Skerfving S. Inorganic lead. In: Beije B, Lundberg P, editors. Criteria documents from the Nordic Expert Group 1992. Stockholm: Arbete och Hälsa; 1993. p. 125-238.

7. Moreira FR, Moreira JC. Os efeitos do chumbo sobre o organismo humano e seu significado para a saúde. Rev Panam Salud Pública 2004; 15:119-29.

8. Sakai T. Biomarkers of lead exposure. Ind Health 2000; 38:127-42.

9. Moreira FR, Moreira JC. A importância da análise de especiação do chumbo em plasma para a avaliação dos riscos à saúde. Química Nova 2004; 27:251-60.

10. Sakai T, Ushio K, Ikeya Y. Mobilized plasma lead as an index of body burden and its relation to the heme-related indices. Ind Health 1998; 36:240-6.

11. Yapici G, Can G, Kiziler AR, Aydemir B, Timur IH, Kaypmaz A. Lead and cadmium exposure in children living around a coal-mining area in Yataðan, Turkey. Toxicol Ind Health 2006; 22:357-62.

12. Afridi HI, Kazi TG, Jamali MK, Kazi GH, Arain MB, Jalbani N, et al. Evaluation of toxic metals in biological samples (scalp hair, blood and urine) of steel mill workers by electrothermal atomic absorption spectrometry. Toxicol Ind Health 2006; 22:381-93.

13. Dos Santos CR, Rodrigues RS, Silva CS, Nascimento ES. Determination of lead in whole blood by electrothermal atomic absorption spectrometry using Zeeman correction and sample stability. Journal of Radioanalytical and Nuclear Chemistry 2006; 269:481-5.

14. Riddell TJ, Solon O, Quimbo SA, Tan CM, Butrick E, Peabody JW. Elevated blood-lead levels among children living in the rural Philippines. Bull World Health Organ 2007; 85:674-80.

15. Dogu O, Louis ED, Tamer L, Unal O, Yilmaz A, Kaleagasi H. Elevated blood lead concentrations in essential tremor: a case-control study in Mersin, Turkey. Environ Health Perspect 2007; 115:1564-8.
16. Schutz A, Olsson M, Jensen A, Gerhardsson L, Borjesson J, Mattsson S, et al. Lead in finger bone, whole blood, plasma and urine in lead-smelter workers: extended exposure range. Int Arch Occup Environ Health 2005; 78:35-43.

17. Gulson BL, Cameron MA, Smith AJ, Mizon KJ, Korsch MJ, Vimpani G, et al. Blood lead-urine lead relationships in adults and children. Environ Res 1998; 78:152-60.

18. Fukui Y, Miki M, Ukai H, Okamoto S, Takada S, Higashikawa $\mathrm{K}$, et al. Urinary lead as a possible surrogate of blood lead among workers occupationally exposed to lead. Int Arch Occup Environ Health 1999; 72:516-20.

19. Shimbo S, Zhang ZW, Moon CS, Watanabe T, Nakatsuka H, Matsuda-Inoguchi N, et al. Correlation between urine and blood concentrations, and dietary intake of cadmium and lead among women in the general population of Japan. Int Arch Occup Environ Health 2000; 73:163-70.

20. Higashikawa K, Zhang ZW, Shimbo S, Moon CS, Watanabe T, Nakatsuka $\mathrm{H}$, et al. Correlation between concentration in urine and in blood of cadmium and lead among women in Asia. Sci Total Environ 2000; 246:97-107.

21. Hirata M, Yoshida T, Miyajima K, Kosaka H, Tabuchi T. Correlation between lead in plasma and other indicators of lead exposure among lead-exposed workers. Int Arch Occup Environ Health 1995; 68:58-63.

22. Moon CS, Zhang ZW, Shimbo S, Watanabe T, Moon $\mathrm{DH}$, Lee CU, et al. Evaluation of urinary cadmium and lead as markers of background exposure of middle-aged women in Korea. Int Arch Occup Environ Health 1998; 71:251-6.

23. Bergdahl IA, Schutz A, Gerhardsson L, Jensen A, Skerfving S. Lead concentrations in human plasma, urine and whole blood. Scand J Work Environ Health 1997; 23:359-63.

24. Tsaih SW, Schwartz J, Lee MLT, Amarasiriwardena C, Aro A, Sparrow D, et al. The independent contribution of bone and erythrocyte lead to urinary lead among middle-aged and elderly men: the normative aging study. Environ Health Perspect 1999; 107:391-6.

25. Minoia C, Sabbioni E, Apostoli P, Pietra R, Pozzoli L, Gallorini M, et al. Trace element reference values in tissues from inhabitants of the European community. I. A study of 46 elements in urine, blood and serum of Italian subjects. Sci Total Environ 1990; 95:89-105.

26. Sackett DL, Haynes RB, Tugwell P. Clinical epidemiology: a basic science for clinical medicine. $2^{\text {nd }}$ Ed. Boston: Little, Brown and Company; 1991.

Recebido em 04/Jul/2007

Versão final reapresentada em 30/Jan/2008

Aprovado em 25/Fev/2008 